\title{
Error estimates for phase recovering from phaseless scattering data *
}

\author{
R.G. Novikov ${ }^{\dagger} \quad$ V.N. Sivkin ${ }^{\ddagger}$
}

November 15, 2019

\begin{abstract}
We study the simplest explicit formulas for approximate finding the complex scattering amplitude from modulus of the scattering wave function. We obtain detailed error estimates for these formulas in dimensions $\mathrm{d}=3$ and $\mathrm{d}=2$.
\end{abstract}

\section{Introduction}

We consider the Schrödinger equation

$$
\begin{gathered}
-\Delta \psi+v(x) \psi=E \psi, \quad x \in R^{d}, d \geq 1, E>0, \\
v \in L_{\infty}\left(R^{d}\right), \text { supp } v \subset D, \\
D \text { is an open bounded domain in } R^{d} .
\end{gathered}
$$

Here $\Delta$ is the standart Laplacian in $x, v$ is a scalar potential.

For equation (1) we consider the classical scattering solutions $\psi^{+}$specified by the following asymptotics as $|x| \rightarrow \infty$ :

$$
\psi^{+}(x, k)=e^{i k x}+c(d,|k|) \frac{e^{i|k||x|}}{|x|^{(d-1) / 2}} f\left(k,|k| \frac{x}{|x|}\right)+O\left(\frac{1}{|x|^{(d+1) / 2}}\right),
$$

$c(d,|k|)=-\pi i(-2 \pi i)^{(d-1) / 2}|k|^{(d-3) / 2}$, for $\sqrt{-2 \pi i}=\sqrt{2 \pi} e^{-i \pi / 4}, x, k \in R^{d}, k^{2}=E$,

where a priori unknown function $f=f(k, l), k, l \in R^{d}, k^{2}=l^{2}=E$, arising in (3) is the classical scattering amplitude for (1).

In order to study $\psi^{+}$and $f$ one can use the Lippmann-Schwinger integral equation (4) and formula (6):

${ }^{*}$ The work was fulfilled during the stage of the second author in the Centre de Mathématique Appliquées of Ecole Polytechnique in September-December 2019.

${ }^{\dagger}$ CMAP, Ecole Polytechnique, CNRS, Université Paris-Saclay, 91128 Palaiseau, France; and IEPT RAS, 117997 Moscow, Russia(novikov@cmap.polytechnique.fr)

†omonosov Moscow State University, Faculty of Mechanics and Mathematics, Moscow, Russia (sivkin96@yandex.ru) 


$$
\begin{gathered}
\psi^{+}(x, k)=e^{i k x}+\int_{R^{d}} G^{+}(x-y, k) v(y) \psi^{+}(y, k) d y \\
G^{+}(x, k):=-\frac{1}{(2 \pi)^{d}} \int_{R^{d}} \frac{e^{i \xi x} d \xi}{\xi^{2}-k^{2}-i 0}=G_{0}^{+}(|x|,|k|), \\
f(k, l)=\frac{1}{(2 \pi)^{d}} \int_{R^{d}} e^{-i l x} v(x) \psi^{+}(x, k) d x,
\end{gathered}
$$

where $x, k, l \in R^{d}, k^{2}=l^{2}=E$, and $G_{0}^{+}$also depends on $d$. Note that:

$$
G^{+}(x, k)=-\frac{i}{4} H_{0}^{1}(|x||k|) \text { for } d=2, G^{+}(x, k)=-\frac{e^{i|k||x|}}{4 \pi|x|} \text { for } d=3,
$$

where $H_{0}^{1}$ is the Hankel function of the first type.

In the present work we also assume that

equation (4) is uniquely solvable for $\psi^{+}(\cdot, k) \in L^{\infty}\left(R^{d}\right)$ for fixed $E>0$,

where $k \in R^{d}, k^{2}=E$. For example, for real-valued $v$ satisfying (2) it holds true.

We recall that $\psi^{+}$describes scattering of the incident plane waves described by $e^{i k x}$ on the scatterer described by $v$. In addition, the second term on the right-hand side of (3) describes the leading scattered spherical waves.

We also recall that in quantum mechanics the values of scatttering functions $\psi^{+}(x, k)$ and $f(k, l)$ have no direct physical sense, whereas the phaseless values $\left|\psi^{+}(x, k)\right|^{2}$ and $|f|^{2}$ have probabilistic interpretation (the Born's principle) and can be obtained in experiments; see [B, FM].

We consider the following problems:

Problem 1. Find $v$ on $R^{d}$ from $f=f(k, l)$ given for appropriate pairs $(k, l)$.

Problem 2. Find $f(k, l)$ from $\left|\psi^{+}(x, k)\right|^{2}$ at appropriate points $x$ such that $x \in R^{d} \backslash D$ and $x /|x|=l /|l|$.

Problem 3. Find $v$ on $R^{d}$ from $\left|\psi^{+}\right|^{2}$ appropriately given outside of $D$.

Problem 1 is the classical inverse scattering problem. This problem was studied in many works; see, for example, [ChS], [E], [M], [N1] and references therein.

Problem 2 is a problem of phase recovering. Note that finding $f$ considered in this problem and formula (3) also yield approximate finding $\psi^{+}$for large $|x|$. In connection with known results on Problem 2, see [N2]-[N5].

Problem 3 is a problem of inverse scattering without phase information. In connection with known results on this problem, see [JL], [N2]-[N5], [K2].

Actually, in the present work we continue studies on Problem 2 in dimentions $d=3$ and $d=2$. Problem 2 for $d=1$ was solved in [N3]. In addition, results on Problem 1 and Problem 2 admit direct applications to Problem 3.

Note that Problem 2 is one of possible problems of phase recovering and Problem 3 is one of possible problems of inverse scattering without phase information. In 
connection with results given on other inverse wave propagation problems without phase information, see [ChS], [IK], [K1], [N2], [N4], [KR], [R], [AHN], [HN], [P] and references therein.

We recall that Problem 2 can be solved approximately by the following explicit formulas of [N2], [N3]:

$$
\begin{aligned}
& \left(\begin{array}{l}
\operatorname{Re} c(d,|k|) f(k, l) \\
\operatorname{Im} c(d,|k|) f(k, l)
\end{array}\right)=M\left(\left(\begin{array}{c}
a\left(x_{1}, k\right) \\
a\left(x_{2}, k\right)
\end{array}\right)-\left(\begin{array}{l}
\delta a\left(x_{1}, k\right) \\
\delta a\left(x_{2}, k\right)
\end{array}\right)\right), \\
& \text { where } \\
& a(x, k)=|x|^{(d-1) / 2}\left(\left|\psi^{+}(x, k)\right|^{2}-1\right), \\
& M=\frac{1}{2 \sin \left(\phi_{2}-\phi_{1}\right)}\left(\begin{array}{cc}
\sin \left(\varphi_{2}\right) & -\sin \left(\varphi_{1}\right) \\
-\cos \left(\varphi_{1}\right) & \cos \left(\varphi_{2}\right)
\end{array}\right), \\
& x_{1}=s \hat{l}, x_{2}=(s+\tau) \hat{l}, \hat{l}=l /|l| \text {, } \\
& \varphi_{j}=|k|\left|x_{j}\right|-k x_{j}, j=1,2 \text {, } \\
& \varphi_{2}-\varphi_{1}=\tau(|k|-k \hat{l}) \text {, } \\
& \delta a\left(x_{1}, k\right)=O\left(s^{-\sigma}\right), \delta a\left(x_{2}, k\right)=O\left(s^{-\sigma}\right) \text { as } s \rightarrow+\infty \text {, }
\end{aligned}
$$

uniformly in $\hat{k}=k /|k|, \hat{l}=l /|l|$ and $\tau$ at fixed $E>0$,

$$
\sigma=1 / 2 \text { for } d=2, \sigma=1 \text { for } d \geq 3,
$$

where $k, l \in R^{d}, k^{2}=l^{2}=E, s>0, \tau>0, \sin \left(\varphi_{1}-\varphi_{2}\right) \neq 0$.

In order to control error in finding $f(k, l)$ from $\left|\psi^{+}(x, k)\right|^{2}$ at $x=x_{1}, x_{2}$ via formulas (9-15) it is necessary to estimate $\delta a(x, k)=O\left(s^{-\sigma}\right)$ in detail. However, detailed estimates for $\delta a(x, k)$ were not yet given in the literature. For the first time such estimates are given in the present work, see Theorem 1 and Lemmas 1 and 2 of Section 2. These estimates are proved in Sections 3-5.

Finally, we recall that $2 n$-point version of formulas (9)-(16) with error term estimated as $O\left(s^{-n}\right), s \rightarrow+\infty$, is given in [N5]. Detailed estimates of this $O\left(s^{-n}\right)$ generalizing the estimates (20)-(23) (see Section 2) of the present work will be given elsewhere.

\section{Main results}

Let

$$
D \subset B_{r}=\left\{x \in R^{d}:|x| \leq r\right\}
$$

for some fixed $r>0$. 
Theorem 1. (A) Under assumptions (2),(8),(19) for $d=3$, the following estimate holds, for $|x| \geq 3 r$ :

$$
\begin{aligned}
& |\delta a(x, k)| \leq \rho_{3} \frac{\left\|\psi^{+}\right\|_{\infty}\|v\|_{L_{1}}}{2 \pi|x|}+\left(1+\frac{2 \rho_{3}}{|x|}+\frac{\rho_{3}^{2}}{|x|^{2}}\right) \frac{\left\|\psi^{+}\right\|_{\infty}^{2}\|v\|_{L_{1}}^{2}}{16 \pi^{2}|x|}, \\
& \rho_{3}:=r\left(4.5+7.65|k| r+3.91|k|^{2} r^{2}\right),
\end{aligned}
$$

where $k, x \in R^{3}, k^{2}=E>0$.

(B) Under assumptions (2),(8),(19) for $d=2$, the following estimate holds:

$$
\begin{aligned}
& |\delta a(x, k)| \leq\left(1+\frac{\rho_{2} \sqrt{2}}{|x|}+\frac{\rho_{2}^{2}}{2|x|^{2}}\right) \frac{\left\|\psi^{+}\right\|_{\infty}^{2}\|v\|_{L_{1}}^{2}}{8 \pi|x|^{1 / 2}|k|}+\rho_{2} \frac{\left\|\psi^{+}\right\|_{\infty}\|v\|_{L_{1}}}{2 \sqrt{\pi}|k|^{1 / 2}|x|}, \\
& \rho_{2}:=r\left(\frac{0.33}{|k| r}+2.51+5.36|k| r+2.14|k|^{2} r^{2}\right)
\end{aligned}
$$

where $k, x \in R^{2}, k^{2}=E>0$.

In Theorem 1 we use the notation $\left\|\psi^{+}\right\|_{\infty}:=\left\|\psi^{+}(\cdot, k)\right\|_{L_{\infty}(D)}$.

Theorem 1 is proved in Sections 3, 4 .

In addition, $\left\|\psi^{+}\right\|_{\infty}$ is estimated in Lemmas 1 and 2 given below.

Let

$$
Q=C_{0}(d, s) \sup _{x \in D}\left|\left(1+|x|^{2}\right)^{s} v(x)\right||k|^{-1},
$$

where $C_{0}$ is the constant of the Agmon estimate (99) (see Section 5).

Lemma 1. (A) Under assumptions (2), (19), for $Q<1$ and $d=3$, the following estimate holds:

$$
\left\|\psi^{+}(\cdot, k)\right\|_{L_{\infty}\left(R^{3}\right)} \leq 1+\sqrt{\frac{5}{6}} \frac{\|v\|_{L_{\infty}(D)}\left(1+r^{2}\right)^{s / 2} r^{1 / 2}}{(1-Q)},
$$

where $s=(d+\varepsilon) / 2, \varepsilon>0,|k| \geq 1$.

(B) Under assumptions (2), (19), for $Q<1$ and $d=2$, the following estimate holds:

$$
\left\|\psi^{+}(\cdot, k)\right\|_{L_{\infty}\left(R^{2}\right)} \leq 1+\frac{\sqrt{\pi}\|v\|_{L_{\infty}(D)}\left(1+r^{2}\right)^{s / 2} r^{1 / 2}}{\sqrt{2 \varepsilon|k|}(1-Q)},
$$

where $s=(d+\varepsilon) / 2, \varepsilon>0,|k| \geq 1$.

Lemma 2. (A) Under assumptions (2), (19), for $\|v\|_{L_{\infty}(D)} r^{2} \leq 2, d=3$ the following estimate holds:

$$
\left\|\psi^{+}\right\|_{L_{\infty}\left(R^{3}\right)} \leq \frac{1}{1-\|v\|_{L_{\infty}(D)} r^{2} / 2} .
$$


(B) Under assumptions (2), (19), for $\sqrt{\frac{2 \pi}{|k|} \frac{\|v\|_{L_{\infty}}(D) r^{5 / 2}}{5}}<1, d=2$ the following estimate holds:

$$
\left\|\psi^{+}\right\|_{L_{\infty}\left(R^{2}\right)} \leq \frac{1}{1-\sqrt{\frac{2 \pi}{|k|} \frac{\|v\|_{L_{\infty}(D)} r^{3 / 2}}{3}}} .
$$

Lemmas 1 and 2 are proved in Section 5.

\section{Proof of Theorem 1 (A)}

We have that (see [N2]):

$$
|\delta a(x, k)|=|x|^{-\frac{d-1}{2}}|c|^{2}|f|^{2}+2|x|^{\frac{d-1}{2}} \operatorname{Re}\left(\delta \psi^{+}(x, k) \overline{\psi_{1}^{+}(x, k)}\right)+|x|^{\frac{d-1}{2}}\left|\delta \psi^{+}(x, k)\right|^{2},
$$

where $f=f\left(k, \frac{|k| x}{|x|}\right)$,

$$
\begin{gathered}
\psi_{1}^{+}(x, k):=e^{i k x}+c(d,|k|) \frac{e^{i|k||x|}}{|x|^{(d-1) / 2}} f\left(k,|k| \frac{x}{|x|}\right), \\
\delta \psi^{+}(x, k):=\psi^{+}(x, k)-\psi_{1}^{+}(x, k) .
\end{gathered}
$$

Note that

$$
|f|=\frac{1}{(2 \pi)^{d}}\left|\int_{R^{d}} e^{-i l x} v(x) \psi^{+}(x, k) d x\right| \leq \frac{1}{(2 \pi)^{d}}\left\|\psi^{+}\right\|_{\infty}\|v\|_{L_{1}} .
$$

Further in this section we always assume that $d=3$.

The following estimate holds:

$$
|\delta a(x, k)| \leq|x|^{-1}\left(2 \pi^{2}\right)^{2}|f|^{2}+2|x|\left|\delta \psi^{+}(x, k)\right|\left|1+\frac{2 \pi^{2}|f|}{|x|}\right|+|x|\left|\delta \psi^{+}(x, k)\right|^{2} .
$$

In addition, $\left|\delta \psi^{+}(x, k)\right|$ is estimated in the following lemma:

Lemma 3. assumptions of Theorem 1A, the following estimate holds, for $|x| \geq 3 r$ :

$$
\left|\delta \psi^{+}(x, k)\right| \leq \frac{\left\|\psi^{+}\right\|_{\infty}\|v\|_{L_{1}}}{4 \pi|x|^{2}} \rho_{3}(r, k) .
$$

Using estimates (29), (32), (34) we have that:

$$
\begin{gathered}
|\delta a(x, k)| \leq|x|^{-1}\left(2 \pi^{2}\right)^{2} \frac{1}{\left((2 \pi)^{3}\right)^{2}}\left\|\psi^{+}\right\|_{\infty}^{2}\|v\|_{L_{1}}^{2}+ \\
+2|x| \frac{1}{4 \pi}\left\|\psi^{+}\right\|_{\infty}\|v\|_{L_{1}} \frac{\rho_{3}}{|x|^{2}}\left(1+\frac{2 \pi^{2} \frac{1}{(2 \pi)^{3}}\left\|\psi^{+}\right\|_{\infty}\|v\|_{L_{1}}}{|x|}\right)+|x|\left(\frac{1}{4 \pi}\left\|\psi^{+}\right\|_{\infty}\|v\|_{L_{1}} \frac{\rho_{3}}{|x|^{2}}\right)^{2} \leq
\end{gathered}
$$




$$
\begin{gathered}
\leq \frac{\left\|\psi^{+}\right\|_{\infty}^{2}\|v\|_{L_{1}}^{2}}{16 \pi^{2}|x|}+\left\|\psi^{+}\right\|_{\infty}\|v\|_{L_{1}} \frac{\rho_{3}}{2 \pi|x|}\left(1+\frac{\left\|\psi^{+}\right\|_{\infty}\|v\|_{L_{1}}}{4 \pi|x|}\right)+ \\
+\left\|\psi^{+}\right\|_{\infty}^{2}\|v\|_{L_{1}}^{2} \frac{\rho_{3}^{2}}{16 \pi^{2}|x|^{3}},|x| \geq 3 r
\end{gathered}
$$

Estimate (20) of Theorem 1A follows from (35). Therefore, in order to prove Theorem 1A it remains to prove Lemma 3.

Proof of Lemma 3. Using the Lippmann-Schwinger integral equation (4) and formulas (6), (31) we obtain

$$
\begin{gathered}
\delta \psi^{+}(x, k)=-\int_{R^{3}} \frac{e^{i|k||x-y|}}{4 \pi|x-y|} v(y) \psi^{+}(y, k) d y+ \\
+2 \pi^{2} \frac{e^{i|k||x|}}{|x|} \frac{1}{(2 \pi)^{3}} \int_{R^{3}} e^{-i|k| \frac{x y}{x \mid}} v(y) \psi^{+}(y, k) d y= \\
=\frac{1}{4 \pi} \int_{R^{3}}\left(\frac{e^{i|k||x|-i|k| \frac{x y}{|x|}}}{|x|}-\frac{e^{i|k||x-y|}}{|x-y|}\right) v(y) \psi^{+}(y, k) d y .
\end{gathered}
$$

From (36) we obtain (37):

$$
\left|\delta \psi^{+}(x, k)\right| \leq \frac{1}{4 \pi}\left\|\psi^{+}(\cdot, k)\right\|_{\infty} \int_{R^{3}}\left|\frac{e^{i|k||x|-i|k| \frac{x y}{|x|}}}{|x|}-\frac{e^{i|k||x-y|}}{|x-y|}\right| v(y) \mid d y .
$$

Lemma 4. Let $x, y \in R^{3},|y| \leq r,|x| \geq 3 r$. Then:

$$
\left|\frac{e^{i|k||x|-i|k| \frac{x y}{|x|}}}{|x|}-\frac{e^{i|k||x-y|}}{|x-y|}\right| \leq \frac{r}{|x|^{2}}\left(4.5+7.65|k| r+3.91|k|^{2} r^{2}\right) .
$$

Estimate (34) of Lemma 3 follows from estimates (37), (38). Thus, in order to prove Lemma 3, it remains to prove Lemma 4.

Proof of Lemma 4. To prove Lemma 4 we use in particular Lemma 5.

Lemma 5. Let $x, y \in R^{3},|y| \leq r,|x| \geq 3 r$. Then the following estimates hold:

$$
\begin{gathered}
|x-y|=|x|\left(1-\frac{x y}{|x|^{2}}+\frac{|y|^{2}}{2|x|^{2}}-\frac{(x y)^{2}}{2|x|^{4}}+L_{3}(x, y)\right),\left|L_{3}(x, y)\right| \leq \frac{4.13 r^{3}}{|x|^{3}} \\
|x-y|=|x|\left(1-\frac{x y}{|x|^{2}}+L_{2}(x, y)\right),\left|L_{2}(x, y)\right| \leq \frac{2.38 r^{2}}{|x|^{2}}
\end{gathered}
$$


Proof of Lemma 5. Recall that

$$
(1+\varepsilon)^{1 / 2}=\sum_{n=0}^{\infty} \frac{(-1)^{n}(2 n) !}{(1-2 n) n !^{2} 4^{n}} \varepsilon^{n}=\sum_{n=0}^{\infty} a_{n} \varepsilon^{n}, \forall|\varepsilon|<1 .
$$

Note that

$$
k_{n+1}:=\frac{a_{n+1}}{a_{n}}=\frac{(-1)(2 n+1)(2 n+2)(1-2 n)}{(n+1)(n+1) 4(1-2 n-2)}=-\frac{2(2 n+1)(2 n-1)}{4(2 n+1)(n+1)}=-\left(1-\frac{3}{2 n+2}\right)
$$

$\left|k_{n+1}\right|<1$, for $n \in N \cup\{0\}$.

Therefore,

$$
\begin{aligned}
& \left|\sum_{n=3}^{\infty} a_{n} \varepsilon^{n}\right| \leq\left|a_{3}\right| \sum_{n=3}^{\infty}\left|\varepsilon^{n}\right| \leq\left|a_{3}\right| \frac{|\varepsilon|^{3}}{1-|\varepsilon|},|\varepsilon|<1, \\
& \left|a_{3}\right|=\frac{6 !}{5 * 3 ! 3 ! 4^{3}}=\frac{6 * 5 * 4 * 6}{5 * 6 * 6 * 4^{3}}=\frac{1}{16} .
\end{aligned}
$$

In the present work we use formulas $(41),(43)$ for

$$
\varepsilon=-\frac{2 x y}{|x|^{2}}+\frac{|y|^{2}}{|x|^{2}}, x, y \in R^{d},|y| \leq r,|x| \geq 3 r .
$$

From (44) it follows that:

$$
|\varepsilon| \leq \frac{7}{9},|\varepsilon| \leq \frac{7}{3} \frac{r}{|x|}
$$

Using (41),(44) we have that

$$
\begin{aligned}
& |x-y|=|x|\left|\frac{x}{|x|}-\frac{y}{|x|}\right|=|x|\left(1-\frac{2 x y}{|x|^{2}}+\frac{|y|^{2}}{|x|^{2}}\right)^{\frac{1}{2}}= \\
& =|x|\left(1-\frac{x y}{|x|^{2}}+\frac{|y|^{2}}{2|x|^{2}}-\frac{1}{8}\left(\frac{|y|^{2}}{|x|^{2}}-\frac{2 x y}{|x|^{2}}\right)^{2}+R_{3}(x, y)\right)
\end{aligned}
$$

where

$$
\left|R_{3}(x, y)\right|=\left|\sum_{n=3}^{\infty} a_{n} \varepsilon^{n}\right| \leq\left|a_{3}\right| \frac{|\varepsilon|^{3}}{1-|\varepsilon|} \leq \frac{1}{16} \frac{7^{3} r^{3}}{3^{3}|x|^{3}} \frac{1}{2 / 9} \leq 3.58 \frac{r^{3}}{|x|^{3}} .
$$

Using (46), (47) and gathering the terms with equal degrees in $|x|$, we obtain:

$$
|x-y|=|x|\left(1-\frac{x y}{|x|^{2}}+\frac{|y|^{2}}{2|x|^{2}}-\frac{(x y)^{2}}{2|x|^{4}}+L_{3}(x, y)\right)
$$




$$
\left|L_{3}(x, y)\right| \leq \frac{1}{8} \frac{|y|^{4}}{|x|^{4}}+\frac{1}{2} \frac{x y|y|^{2}}{|x|^{4}}+R_{3}(x, y) \leq \frac{r^{3}}{24|x|^{3}}+\frac{r^{3}}{2|x|^{3}}+3.58 \frac{r^{3}}{|x|^{3}} \leq \frac{4.13 r^{3}}{|x|^{3}}
$$

Thus, estimate (39) is proved.

In addition to (46), (47) we also need the following formulas:

$$
\begin{gathered}
|x-y|=|x|\left(1-\frac{x y}{|x|^{2}}+\frac{|y|^{2}}{2|x|^{2}}+R_{2}(x, y)\right) \\
\left|R_{2}(x, y)\right|=\left|\sum_{n=2}^{\infty} a_{n} \varepsilon^{n}\right| \leq\left|a_{2} \varepsilon^{2}\right|+R_{3}(x, y) \leq \frac{7^{2} / 3^{2}}{8} \frac{r^{2}}{|x|^{2}}+3.58 \frac{r^{3}}{|x|^{3}} \leq 1.88 \frac{r^{2}}{|x|^{2}} .
\end{gathered}
$$

In a similar way with (48), (49) we have

$$
\begin{gathered}
|x-y|=|x|\left(1-\frac{x y}{|x|^{2}}+L_{2}(x, y)\right), \\
\left|L_{2}(x, y)\right| \leq \frac{r^{2}}{2|x|^{2}}+1.88 \frac{r^{2}}{|x|^{2}} \leq \frac{2.38 r^{2}}{|x|^{2}} .
\end{gathered}
$$

Thus, estimate (40) is proved. This completes the prove of Lemma 5.

Now we are ready to prove estimate (38). We have

$$
\begin{aligned}
& \left|\frac{e^{i|k||x|-i|k| \frac{x y}{|x|}}}{|x|}-\frac{e^{i|k||x-y|}}{|x-y|}\right|= \\
& =\left|\frac{e^{i|k||x|-i|k| \frac{x y}{|x|}}}{|x|}-\frac{e^{i|k||x|\left(1-\frac{x y}{|x|^{2}}+\frac{|y|^{2}}{2|x|^{2}}-\frac{(x y)^{2}}{2|x|^{4}}+L_{3}(x, y)\right)}}{|x|\left(1-\frac{x y}{|x|^{2}}+L_{2}(x, y)\right)}\right|= \\
& =\frac{1}{|x|}\left|1-\frac{e^{i|k||x|\left(\frac{|y|^{2}}{2|x|^{2}}-\frac{(x y)^{2}}{2|x|^{4}}+L_{3}(x, y)\right)}}{1-\frac{x y}{|x|^{2}}+L_{2}(x, y)}\right|=: \frac{1}{|x|}\left|1-\frac{e^{i L}}{1-t}\right| .
\end{aligned}
$$

Note that

$$
\left|e^{i L}-1-i L\right| \leq \frac{L^{2}}{2}, \text { for } L \in R, \text { and }\left|\frac{1}{1-t}-1-t\right| \leq t^{2} \frac{1}{1-|t|},|t|<1,
$$

and further

$$
\begin{gathered}
\left|1-\frac{e^{i L}}{1-t}\right| \leq\left|1-\left(1+L+L^{2} / 2\right)\left(1+t+t^{2} /(1-|t|)\right)\right| \leq \\
\quad \leq L+L^{2} / 2+t+\frac{t^{2}}{1-|t|}+\left(L+L^{2} / 2\right)\left(t+t^{2} /(1-|t|)\right) .
\end{gathered}
$$


In our case:

$$
\begin{gathered}
|L|=|k||x|\left|\frac{|y|^{2}}{2|x|^{2}}-\frac{(x y)^{2}}{2|x|^{4}}+L_{3}(x, y)\right| \leq \frac{|k|}{|x|}\left(\frac{r^{2}}{2}+\frac{r^{2}}{2}+\frac{4.13 r^{3}}{|x|}\right) \leq \frac{2.38|k| r^{2}}{|x|} \\
L^{2} / 2 \leq \frac{2.38^{2}|k|^{2} r^{4}}{2|x|^{2}} \\
|t|=\left|\frac{x y}{|x|^{2}}-L_{2}(x, y)\right| \leq \frac{r}{|x|}+\frac{2.38 r^{2}}{|x|^{2}} \leq \frac{r}{|x|}+\frac{0.8 r}{|x|} \leq \frac{1.8 r}{|x|} \\
\frac{t^{2}}{1-t} \leq \frac{\frac{1.8^{2} r^{2}}{|x|^{2}}}{1-1.8 / 3}=\frac{1.8^{2} r^{2}}{|x|^{2}} \frac{1}{0.4}=8.1 \frac{r^{2}}{|x|^{2}}
\end{gathered}
$$

Finally,$$
\left|1-\frac{e^{i L}}{1-t}\right| \leq \frac{2.38|k| r^{2}}{|x|}+\frac{2.38^{2}|k|^{2} r^{4}}{2|x|^{2}}+\frac{1.8 r}{|x|}+\frac{8.1 r^{2}}{|x|^{2}}+
$$$$
+\left(\frac{2.38|k| r^{2}}{|x|}+\frac{2.38^{2}|k|^{2} r^{4}}{2|x|^{2}}\right)\left(\frac{1.8 r}{|x|}+\frac{8.1 r^{2}}{|x|^{2}}\right) \leq
$$$$
\leq \frac{3.06|k| r^{2}}{|x|}+\frac{3.06^{2}|k|^{2} r^{4}}{2|x|^{2}}+\frac{1.8 r}{|x|}+\frac{8.1 r^{2}}{|x|^{2}}+1.5\left(\frac{3.06|k| r^{2}}{|x|}+\frac{3.06^{2}|k|^{2} r^{4}}{2|x|^{2}}\right) \leq
$$$$
\leq \frac{r}{|x|}\left(4.5+7.65|k| r+3.91|k|^{2} r^{2}\right) \text {. }
$$

Estimate (38) follows from (54), (58), that proves Lemma 4. $\square$.

This also completes the proof of Theorem 1A. $\square$.

\section{Proof of the Theorem 1(B)}

Proceeding from (29)-(31), for $d=2$, we have:

$$
|\delta a(x, k)| \leq|x|^{-1 / 2}|c|^{2}|f|^{2}+2|x|^{1 / 2}\left|\delta \psi^{+}(x, k)\right|\left(1+\frac{|c||f|}{|x|^{1 / 2}}\right)+|x|^{1 / 2}\left|\delta \psi^{+}(x, k)\right|^{2}
$$

where

$$
c=c(2,|k|)=-\pi i(-2 \pi i)^{\frac{2-1}{2}}|k|^{\frac{2-3}{2}}=-(1+i) \pi^{3 / 2}|k|^{-1 / 2} .
$$

Now $|f|$ is estimated in (32) for $d=2$ and $\left|\delta \psi^{+}(x, k)\right|$ is estimated in the following lemma: 
Lemma 6. Under the assumptions of Theorem 1B, the following estimate holds for $|x| \geq 3 r$ :

$$
\left|\delta \psi^{+}(x, k)\right| \leq \frac{\|v\|_{L_{1}}\left\|\psi^{+}\right\|_{\infty} \rho_{2}(|k|, r)}{4(\pi|k|)^{1 / 2}|x|^{3 / 2}} .
$$

Using estimates (59), (32), (61) we have that

$$
\begin{aligned}
& |\delta a(x, k)| \leq|x|^{-1 / 2}|k|^{-1} \frac{\left\|\psi^{+}\right\|_{\infty}^{2}\|v\|_{L_{1}}^{2}}{8 \pi}+ \\
& +|x|^{1 / 2} \frac{\|v\|_{L_{1}}\left\|\psi^{+}\right\|_{\infty} \rho_{2}}{2 \sqrt{\pi}|k|^{1 / 2}|x|^{3 / 2}}\left(1+\frac{\left\|\psi^{+}\right\|_{\infty}\|v\|_{L_{1}}}{2|x|^{1 / 2}(2 \pi)^{1 / 2}|k|^{1 / 2}}\right)+|x|^{1 / 2} \frac{\|v\|_{L_{1}}^{2}\left\|\psi^{+}\right\|_{\infty}^{2} \rho_{2}^{2}}{16 \pi|k||x|^{3}}
\end{aligned}
$$

Estimate (22) of Theorem 1B follows from (62). Therefore, in order to prove Theorem 1B it remains to prove Lemma 6.

Proof of Lemma 6. Using the Lippmann-Schwinger integral equation (4) and formulas (6), (31), (7) we obtain

$$
\begin{gathered}
\delta \psi^{+}(x, k)=\int_{R^{2}}-\frac{i H_{0}^{(1)}(|k||x-y|)}{4} v(y) \psi^{+}(y, k) d y+ \\
+(1+i) \frac{\pi^{3 / 2}}{|k|^{1 / 2}} \frac{e^{i|k||x|}}{|x|^{1 / 2}} \frac{1}{(2 \pi)^{2}} \int_{R^{2}} e^{-i|k| \frac{x y}{|x|}} v(y) \psi^{+}(y, k) d y= \\
=\int_{R^{2}}\left(-\frac{i H_{0}^{(1)}(|k||x-y|)}{4}+\frac{(1+i) e^{i|k||x|-i|k| \frac{x y}{|x|}}}{4(\pi|k||x|)^{1 / 2}}\right) v(y) \psi^{+}(y, k) d y .
\end{gathered}
$$

From (64) we obtain (65):

$$
\left|\delta \psi^{+}(x, k)\right| \leq\left\|\psi^{+}(\cdot, k)\right\|_{\infty} \int_{R^{2}}\left|-\frac{i H_{0}^{(1)}(|k||x-y|)}{4}+\frac{(1+i) e^{i|k||x|-i|k| \frac{x y}{|x|}}}{4(\pi|k||x|)^{1 / 2}}\right||v(y)| d y .
$$

Lemma 7. Let $x, y \in R^{2},|y| \leq r,|x| \geq 3 r$. Then

$$
\begin{gathered}
\left|-\frac{i H_{0}^{(1)}(|k||x-y|)}{4}+\frac{(1+i) e^{i|k||x|-i|k| \frac{x y}{|x|}}}{4(\pi|k||x|)^{1 / 2}}\right|=M_{1}+M_{2} \text {, where } \\
M_{1} \leq \frac{\sqrt{2}}{4(\pi k)^{1 / 2}|x|^{3 / 2}}\left(1.77+3.79|k| r+1.51|k|^{2} r^{2}\right) ; \\
M_{2} \leq \frac{3 \sqrt{3}}{64(\pi|k||x|)^{1 / 2}|k||x|} .
\end{gathered}
$$


Note that

$$
M_{1}+M_{2} \leq \frac{\sqrt{2} r}{4(\pi|k|)^{1 / 2}|x|^{3 / 2}}\left(\frac{3 \sqrt{3}}{16 \sqrt{2}|k| r}+1.77+3.79|k| r+1.51|k|^{2} r^{2}\right) .
$$

Estimate (61) of Lemma 6 follows from (65)-(69). Thus, in order to prove Lemma 6 it remains to prove Lemma 7.

Proof of Lemma \%. To prove Lemma 7 we use, in particular, estimate (39) of Lemma 5 , which remains the same for $d=2$. Besides, we use, in particular, Lemma 8.

Lemma 8. For Hankel funtion the following representation holds:

$$
H_{0}^{(1)}(|k||x|)=\left(\frac{2}{\pi|k||x|}\right)^{1 / 2} e^{i|k||x|-i \pi / 4}(1+h(|k||x|)), \quad|h(|k||x|)| \leq \frac{1}{8|k||x|} .
$$

Lemma 8 is proved at the end of this Section.

Using formula (70) we have

$$
\begin{aligned}
& \left|-\frac{i H_{0}^{(1)}(|k||x-y|)}{4}+\frac{(1+i) e^{i|k||x|-i|k| \frac{x y}{|x|}}}{4(\pi|k||x|)^{1 / 2}}\right| \leq \\
& \left|-\frac{i}{4}\left(\frac{2}{\pi|k||x-y|}\right)^{1 / 2} e^{i|k||x-y|-i \pi / 4}(1+h(|k|(x-y)))+\frac{(1+i) e^{i|k||x|-i|k| \mid \frac{x y}{|x|}}}{4(\pi|k||x|)^{1 / 2}}\right| \leq \\
& \leq \frac{1}{4(\pi|k|)^{1 / 2}}\left|-i \sqrt{2}\left(\frac{\sqrt{2}}{2}-i \frac{\sqrt{2}}{2}\right) \frac{e^{i|k||x-y|}}{|x-y|^{1 / 2}}+(1+i) \frac{e^{i|k||x|-i|k| \frac{x y}{|x|}}}{|x|^{1 / 2}}\right|+ \\
& +\frac{1}{4}\left(\frac{2}{\pi|k||x-y|}\right)^{1 / 2} \frac{1}{8|k||x-y|}=: M_{1}+M_{2} .
\end{aligned}
$$

The term $M_{2}$ can be estimated easily:

$$
M_{2} \leq \frac{3 \sqrt{3}}{64(\pi|k||x|)^{1 / 2}|k||x|},|y| \leq r,|x| \geq 3 r .
$$

Lemma 9. The term $M_{1}$ can be estimated as follows:

$$
M_{1} \leq \frac{\sqrt{2}}{4(\pi|k|)^{1 / 2}|x|^{3 / 2}}\left(1.77+3.79|k| r+1.51|k|^{2} r^{2}\right),|y| \leq r,|x| \geq 3 r .
$$

Proof of Lemma 9. One can see that

$$
M_{1}=\frac{\sqrt{2}}{4(\pi|k|)^{1 / 2}}\left|\frac{e^{i|k||x|-i|k| \frac{x y}{|x|}}}{|x|^{1 / 2}}-\frac{e^{i|k||x-y|}}{|x-y|^{1 / 2}}\right| .
$$

Further, we obtain 


$$
\begin{aligned}
& M_{1} \leq \frac{\sqrt{2}}{4(\pi|k|)^{1 / 2}}\left|\frac{e^{i|k||x|-i|k| \frac{x y}{|x|}}}{|x|^{1 / 2}}-\frac{e^{i|k||x|\left(1-\frac{x y}{|x|^{2}}+\frac{|y|^{2}}{2|x|^{2}}-\frac{(x y)^{2}}{\left.2|x|\right|^{4}}+L_{3}(x, y)\right)}}{|x|^{1 / 2}\left(1-\frac{x y}{2|x|^{2}}+\widetilde{L_{2}}(x, y)\right)}\right|= \\
& =\frac{\sqrt{2}}{4(\pi|k||x|)^{1 / 2}}\left|1-\frac{e^{i|k||x|\left(\frac{|y|^{2}}{2|x|^{2}}-\frac{(x y)^{2}}{2|x|^{4}}+L_{3}(x, y)\right)}}{\left(1-\frac{x y}{2|x|^{2}}+\widetilde{L_{2}}(x, y)\right)}\right| .
\end{aligned}
$$

Here we use the same expantion as in (39) and the following additional expantion:

$$
|x-y|^{1 / 2}=|x|^{1 / 2}\left(1-\frac{2 x y}{|x|^{2}}+\frac{|y|^{2}}{|x|^{2}}\right)^{1 / 4}=|x|^{1 / 2}\left(1-\frac{x y}{2|x|^{2}}+\widetilde{L_{2}}(x, y)\right) \text {. }
$$

To complete the proof of Lemma 9 we use, in particular, the estimate for $L_{3}$ in (39) and the estimate for $\widetilde{L_{2}}$ given in the following Lemma.

Lemma 10. Let $x, y \in R^{2},|y| \leq r,|x| \geq 3 r$. Then:

$$
\left|\widetilde{L_{2}}(x, y)\right| \leq 1.81 \frac{r^{2}}{|x|^{2}} .
$$

Proof of Lemma 10. Recall that

$$
(1+\varepsilon)^{1 / 4}=\sum_{n=0}^{\infty} b_{n} \varepsilon^{n},|\varepsilon|<1, \text { where } b_{n}=\prod_{k=1}^{n} \frac{1 / 4-k+1}{k} .
$$

Note that

$$
l_{n+1}=\frac{b_{n+1}}{b_{n}}=\frac{1 / 4-n-1+1}{n+1}=(-1)\left(1-\frac{5}{4(n+1)}\right),
$$

$\left|l_{n+1}\right|<1$, for $n \in N \cup\{0\}$.

Therefore,

$$
\begin{gathered}
\left|\sum_{n=2}^{\infty} b_{n} \varepsilon^{n}\right| \leq\left|b_{2}\right| \varepsilon^{2}+\left|b_{3}\right| \frac{|\varepsilon|^{3}}{1-|\varepsilon|}, \\
\left|b_{2}\right|=3 / 32,\left|b_{3}\right|=7 / 128
\end{gathered}
$$

In the present work we use formulas $(78),(79)$ for

$$
\varepsilon=-\frac{2 x y}{|x|^{2}}+\frac{|y|^{2}}{|x|^{2}}, x, y \in R^{2},|y| \leq r,|x| \geq 3 r
$$




$$
|\varepsilon| \leq \frac{7}{9},|\varepsilon| \leq \frac{7}{3} \frac{r}{|x|} .
$$

Using (78), (80) we have that

$$
|x-y|^{1 / 2}=|x|^{1 / 2}\left(1-\frac{2 x y}{|x|^{2}}+\frac{|y|^{2}}{|x|^{2}}\right)^{1 / 4}=|x|^{1 / 2}\left(1-\frac{x y}{2|x|^{2}}+\frac{|y|^{2}}{4|x|^{2}}+\widetilde{R_{2}}(x, y)\right),
$$

where

$$
\begin{aligned}
& \left|\widetilde{R_{2}}(x, y)\right|=\left|\sum_{n=2}^{\infty} b_{n} \varepsilon^{n}\right| \leq\left|b_{2}\right| \varepsilon^{2}+\left|b_{3}\right| \frac{\varepsilon^{3}}{1-|\varepsilon|} \leq \frac{3}{32} \frac{7^{2}}{3^{2}} \frac{r^{2}}{|x|^{2}}+\frac{7}{128} \frac{7^{3}}{3^{3}} \frac{r^{3}}{|x|^{3}} \frac{9}{2} \leq \\
& \leq\left(\frac{7^{2}}{32 * 3}+\frac{7 * 7^{3} * 9}{128 * 3^{3} * 3 * 2}\right) \frac{r^{2}}{|x|^{2}} \leq 1.56 \frac{r^{2}}{|x|^{2}}
\end{aligned}
$$

Using (82), (83) and gathering the terms with equal degrees in $|x|$, we obtain:

$$
\left|\widetilde{L_{2}}(x, y)\right|=\left|\frac{|y|^{2}}{4|x|^{2}}+\widetilde{R_{2}}(x, y)\right| \leq \frac{r^{2}}{4|x|^{2}}+1.56 \frac{r^{2}}{|x|^{2}} \leq 1.81 \frac{r^{2}}{|x|^{2}} .
$$

Lemma 10 is proved.

Returning to the proof of Lemma 9, we rewrite (75) as:

$$
M_{1} \leq \frac{\sqrt{2}}{4(\pi|k| x)^{1 / 2}}\left|1-\frac{e^{i L}}{1-t}\right| .
$$

We have

$$
\begin{aligned}
& \left|1-\frac{e^{i L}}{1-t}\right| \leq\left|1-\left(1+L+L^{2} / 2\right)\left(1+t+t^{2} /(1-t)\right)\right| \leq \\
& \leq L+L^{2} / 2+t+\frac{t^{2}}{1-t}+\left(L+L^{2} / 2\right)\left(t+t^{2} /(1-t)\right) .
\end{aligned}
$$

In addition:

$$
\begin{gathered}
L \leq|k||x|\left(\frac{r^{2}}{2|x|^{2}}+\frac{r^{2}}{2|x|^{2}}+\frac{4.13 r^{3}}{|x|^{3}}\right) \leq \frac{|k| r^{2}}{|x|}\left(1+\frac{4.13}{3}\right) \leq \frac{2.38|k| r^{2}}{|x|} \\
L^{2} / 2 \leq 2.84 \frac{|k|^{2} r^{4}}{|x|^{2}} \\
t \leq \frac{r}{2|x|}+1.81 \frac{r^{2}}{|x|^{2}} \leq 1.11 \frac{r}{|x|} \\
\frac{t^{2}}{1-t} \leq \frac{\left(1.11 \frac{r}{|x|}\right)^{2}}{1-1.11 / 3} \leq 1.9558 \frac{r^{2}}{|x|^{2}}
\end{gathered}
$$




$$
\begin{aligned}
& \left(L+L^{2} / 2\right)\left(t+t^{2} /(1-t)\right) \leq\left(\frac{2.38|k| r^{2}}{|x|}+\frac{2.84|k|^{2} r^{4}}{|x|^{2}}\right)\left(1.11 \frac{r}{|x|}+1.9558 \frac{r^{2}}{|x|^{2}}\right) \leq \\
& \leq 0.59\left(\frac{2.38|k| r^{2}}{|x|}+\frac{2.84|k|^{2} r^{4}}{|x|^{2}}\right) .
\end{aligned}
$$

Using (86)-(91), we obtain:

$$
\begin{aligned}
& \left|1-\frac{e^{i L}}{1-t}\right| \leq \frac{2.38 * 1.59|k| r^{2}}{|x|}+\frac{2.84 * 1.59|k|^{2} r^{4}}{|x|^{2}}+1.77 \frac{r}{|x|} \leq \\
& \leq \frac{r}{|x|}\left(1.77+3.79|k| r+1.51|k|^{2} r^{2}\right) .
\end{aligned}
$$

The estimate (73) follows from (85) and (92). This completes the proof of Lemma 9.

Proof of Lemma 8. For $H_{0}^{1}$ the following equality holds, for $s>0$, see in [S] formula (43) for $\nu=0, \beta=0$ :

$$
H_{0}^{(1)}(s)=\left(\frac{2}{\pi s}\right)^{1 / 2} \frac{e^{i s-i \pi / 4}}{\Gamma(1 / 2)} \int_{0}^{\infty} e^{-u} u^{-1 / 2}\left(1+\frac{i u}{2 s}\right)^{-1 / 2} d u
$$

Then we apply the Teylor's expantion with one term and the integral reminder to the function $g(t)=\left(1+\frac{i u t}{2 s}\right)^{1 / 2}, t=0,1, u, s \in R$. We obtain:

$$
\left(1+\frac{i u}{2 s}\right)^{-1 / 2}=1+\frac{1 / 2}{0 !} \frac{u}{2 i s} \int_{0}^{1}\left(1-\frac{u t}{2 i s}\right)^{-3 / 2} d t
$$

So,

$$
\left|\left(1+\frac{i u}{2 s}\right)^{-1 / 2}\right| \leq 1+\frac{u}{4 s} \int_{0}^{1}\left|1-\frac{u t}{2 i s}\right|^{-3 / 2} d t \leq 1+\frac{u}{4 s} .
$$

Using (93), (95), we obtain:

$$
\left|\frac{H_{0}^{(1)}(s)-\left(\frac{2}{\pi s}\right)^{1 / 2} e^{i s-i \pi / 4}}{\left(\frac{2}{\pi s}\right)^{1 / 2} e^{i s-i \pi / 4}}\right| \leq \frac{1}{4 s \Gamma(1 / 2)} \int_{0}^{\infty} e^{-u} u^{-1 / 2} u d u \leq \frac{\Gamma(3 / 2)}{4 s \Gamma(1 / 2)}=\frac{1}{8 s} .
$$

Estimate (96) implies (70).

\section{$5 \quad$ Estimates for $\left\|\psi^{+}\right\|_{\infty}$}

The Lippmann-Schwinger integral equation (4) can also be rewritten as: 


$$
\left(I-A^{+}(|k|)\right) \varphi^{+}(\cdot, k)=\varphi_{0}^{+}(\cdot, k)
$$

where

$$
\begin{aligned}
& \varphi^{+}(x, k)=\Lambda^{-s} \psi^{+}(x, k), \varphi_{0}^{+}(x, k)=\Lambda^{-s} e^{i k x}, \\
& A^{+}(|k|)=\Lambda^{-s} G^{+}(|k|) \Lambda^{-s}\left(\Lambda^{2 s} v\right),
\end{aligned}
$$

where $I$ is the identity operator, $\Lambda$ denotes the multiplication operator by the functions $\left(1+|x|^{2}\right)^{1 / 2}, G^{+}(|k|)$ denotes the integral operator with the Schwartz kernel $G^{+}(x-y, k)$ of (4), (5), $v$ is the multiplication operator by the function $v(x)$, $s=(d+\varepsilon) / 2, \varepsilon>0, k \in R^{d} \backslash\{0\}, x \in R^{d}$.

We recall that the following Agmon estimate holds:

$$
\left\|\Lambda^{-s} G^{+}(|k|) \Lambda^{-s}\right\|_{L_{2}\left(R^{d}\right) \rightarrow L_{2}\left(R^{d}\right)} \leq C_{0}(d, s)|k|^{-1}, s>1 / 2,|k| \geq 1,
$$

see, for example, [E], [N1] and references therein.

Using (99) one can see that

$$
\begin{gathered}
\left\|A^{+}(|k|)\right\|_{L_{2}\left(R^{d}\right) \rightarrow L_{2}\left(R^{d}\right)} \leq Q, s>1 / 2,|k| \geq 1, \\
Q:=C_{0}(d, s)\left\|\Lambda^{2 s} v\right\|_{\infty}|k|^{-1} .
\end{gathered}
$$

As a corollary of (97), (98), (100), we have that if $Q<1, s>d / 2,|k| \geq 1$, then:

$$
\left\|\varphi^{+}\right\|_{L_{2}\left(R^{d}\right)} \leq \frac{\left\|\varphi_{0}^{+}\right\|_{L_{2}\left(R^{d}\right)}}{1-Q}
$$

where

$$
\left\|\varphi_{0}^{+}\right\|_{L_{2}\left(R^{d}\right)}=\left(\int_{R^{d}} \frac{d x}{\left(1+|x|^{2}\right)^{s}}\right)^{1 / 2}=: I_{d}(s) .
$$

Proof of Lemma 1. Using (97), (98), (102) we obtain that

$$
\begin{gathered}
\left.\left|\psi^{+}(x, k)-e^{i k x}\right| \leq \mid \int_{B_{r}} G^{+}(x-y, k) v(y)<y>^{s} \varphi^{+}(y, k)\right) d y \mid \leq \\
\leq\left\|\varphi^{+}(\cdot, k)\right\|_{L_{2}\left(R^{d}\right)} J(x) \leq \frac{\left\|\varphi_{0}^{+}(\cdot, k)\right\|_{L_{2}}}{1-Q} J(x), \quad x \in R^{d} \\
J(x)=\left(\int_{B_{r}}\left|G^{+}(x-y, k) v(y)<y>^{s}\right|^{2} d y\right)^{1 / 2} .
\end{gathered}
$$


For $d=3$ we have:

$$
\begin{gathered}
\left\|\varphi_{0}^{+}\right\|_{L_{2}\left(R^{3}\right)}^{2} \leq \int_{R^{3}} \frac{d y}{\left(1+|y|^{2}\right)^{s}} \leq 4 \pi \int_{0}^{\infty} \frac{r^{2} d r}{\left(1+r^{2}\right)^{s}} \leq \\
\leq 4 \pi\left(\int_{0}^{1} \frac{r^{2} d r}{\left(1+r^{2}\right)^{s}}+\int_{1}^{\infty} \frac{r^{2} d r}{\left(1+r^{2}\right)^{s}}\right) \leq \\
\leq 4 \pi\left(\frac{1}{3}+\int_{1}^{\infty} \frac{r^{2} d r}{\left(r^{2}\right)^{s}}\right)=4 \pi\left(\frac{1}{3}+\frac{1}{(2 s-1)}\right) \leq \frac{10}{3} \pi \\
J(x)=\left(\int_{B_{r}} \frac{v^{2}(y)\left(1+y^{2}\right)^{s}}{16 \pi^{2}|x-y|^{2}} d y\right)^{1 / 2} \leq \frac{\|v\|_{L_{\infty}(D)}\left(1+r^{2}\right)^{s / 2}}{4 \pi}\left(\int_{B_{r}} \frac{1}{|y|^{2}} d y\right)^{1 / 2} \\
\leq \frac{1}{4 \pi}\|v\|_{L_{\infty}(D)}\left(1+r^{2}\right)^{s / 2}\left\|\left(4 \pi \int_{0}^{r} d \rho\right)^{1 / 2}\right\|=\frac{\|v\|_{L_{\infty}(D)}\left(1+r^{2}\right)^{s / 2} r^{1 / 2}}{2 \sqrt{\pi}}, x \in R^{3} .
\end{gathered}
$$

For $d=2$ we have:

$$
\begin{gathered}
\left\|\varphi_{0}^{+}\right\|_{L_{2}}^{2}=\int_{R^{d}}\left|\varphi_{0}^{+}(y, k)\right|^{2} d y=\int_{R^{2}} \frac{d y}{\left(1+|y|^{2}\right)^{s}}= \\
=\pi \int_{0}^{\infty} \frac{d r^{2}}{\left(1+r^{2}\right)^{s}} \leq \pi \int_{0}^{\infty} \frac{d z}{(1+z)^{s}}=\frac{2 \pi}{\varepsilon} ; \\
J(x)=\left(\int_{B_{r}} \frac{\left|H_{0}^{(1)}(|k||x-y|)\right|^{2}}{16}|v(y)|^{2}\left(1+|y|^{2}\right)^{s} d y\right)^{1 / 2} \leq \\
\leq\|v\|_{L_{\infty}(D)}\left(1+r^{2}\right)^{s / 2} \frac{1}{4}\left(\int_{B_{r}}\left|H_{0}^{(1)}(|k||x-y|)\right|^{2} d y\right)^{1 / 2} .
\end{gathered}
$$

From (93) we obtain:

$$
\left|H_{0}^{(1)}(s)\right| \leq\left(\frac{2}{\pi s}\right)^{1 / 2} \frac{1}{\Gamma(1 / 2)} \int_{0}^{\infty} e^{-u} u^{-1 / 2}\left|1+\frac{i u}{2 s}\right|^{-1 / 2} d u \leq\left(\frac{2}{\pi s}\right)^{1 / 2} .
$$

Hence, we have:

$$
\begin{aligned}
& \left(\int_{B_{r}}\left|H_{0}^{(1)}(|k||x-y|)\right|^{2} d y\right)^{1 / 2} \leq\left(\int_{B_{r}} \frac{2}{\pi|k||x-y|} d y\right)^{1 / 2} \leq \\
& \leq\left(\int_{0}^{r} 2 \pi r \frac{2}{\pi|k| r} d r\right)^{1 / 2}=2 \sqrt{\frac{r}{|k|}}
\end{aligned}
$$

Estimate (25) follows from (104), (106), (107).

Estimate (26) follows from (104), (109), (110), (112).

Lemma 9 is proved. 
Proof of Lemma 2 (A). Using the Lippmann-Schwinger equation (4) for $d=3$, we obtain that

$$
\begin{aligned}
& \left\|\psi^{+}\right\|_{\infty} \leq 1+\left\|\psi^{+}\right\|_{\infty}\|v\|_{\infty} \int_{B_{r}} \frac{d x}{4 \pi|x|} \leq 1+\left\|\psi^{+}\right\|_{\infty}\|v\|_{\infty} \int_{0}^{r} \frac{r^{2}}{r} d r \leq \\
& \leq 1+\left\|\psi^{+}\right\|_{\infty}\|v\|_{\infty} \frac{r^{2}}{2} d r .
\end{aligned}
$$

Estimates (27) follows from (113).

(B). Using the Lippmann-Schwinger equation (4) for $d=2$, we obtain that

$$
\begin{aligned}
& \left\|\psi^{+}\right\|_{\infty} \leq 1+\left\|\psi^{+}\right\|_{\infty}\|v\|_{\infty} \int_{B_{r}} \frac{\left|H_{0}^{1}(|x||k|)\right| d x}{4} \leq 1+\frac{\left\|\psi^{+}\right\|_{\infty}\|v\|_{\infty}}{4} \int_{0}^{r}\left(\frac{2}{\pi|k| r}\right)^{1 / 2} 2 \pi r d r \leq \\
& \leq 1+\left\|\psi^{+}\right\|_{\infty}\|v\|_{\infty} \sqrt{\frac{\pi}{2|k|}} \int_{0}^{r} r^{1 / 2} d r \leq 1+\sqrt{\frac{2 \pi}{|k|}} \frac{\left\|\psi^{+}\right\|_{\infty}\|v\|_{\infty} r^{3 / 2}}{3}
\end{aligned}
$$

Estimates (28) follows from (114).

Lemma 10 is proved.

\section{References}

[AHN] A.D. Agaltsov, T. Hohage, R.G. Novikov, An iterative approach to monochromatic phaseless inverse scattering, Inverse Problems 35(2), 24001 ( 24 pp.) (2019)

[B] M. Born, Quantenmechanik der Stossvorgange, Zeitschrift fur Physik 38 (1112), 803-827(1926)

[ChS] K. Chadan, P.C. Sabatier, Inverse Problems in Quantum Scattering Theory, 2nd edn. Springer, Berlin, 1989.

[E] G. Eskin, Lectures on Linear Partial Differential Equations, Graduate Studies in Mathematics, Vol. 123, American Mathematical Society, 2011.

[FM] L.D. Faddeev, S.P. Merkuriev, Quantum Scattering Theory for Multiparticle Systems, Mathematical Physics and Applied Mathematics, 11. Kluwer Academic Publishers Group, Dordrecht, 1993

[IK] O. Ivanyshyn, R. Kress, Identification of sound-soft 3D obstacles from phaseless data, Inverse Probl. Imaging 4, 131-149 (2010)

[JL] P. Jonas, A.K. Louis, Phase contrast tomography using holographic measurements, Inverse Problems 20(1), 75-102 (2004)

[HN] T. Hohage, R.G. Novikov, Inverse wave propagation problems without phase information, Inverse Problems 35(7), 070301 (4 pp.)(2019)

[K1] M.V. Klibanov, Phaseless inverse scattering problems in three dimensions, SIAM J.Appl. Math. 74(2), 392-410 ( 2014)

[K2] M.V. Klibanov, N.A. Koshev, D.-L. Nguyen, L.H. Nguyen, A. Brettin, V.N. Astratov, A numerical method to solve a phaseless coefficient inverse problem from 
a single measurement of experimental data. SIAM J. Imaging Sci. 11(4), 2339-2367 (2018)

[KR] M.V. Klibanov, V.G. Romanov, Reconstruction procedures for two inverse scattering problems without the phase information, SIAM J. Appl. Math. 76(1), 178-196 (2016)

[M] R.B. Melrose, Geometric scattering theory. Stanford Lectures. Cambridge University Press, 1995.

[N1] R. G. Novikov, An iterative approach to non-overdetermined inverse scattering at fixed energy, Sbornik: Mathematics 206(1), 120-134 (2015)

[N2] R. G. Novikov, Formulas for phase recovering from phaseless scattering data at fixed frequency, Bulletin des Sciences Mathematiques 139(8), 923-936 (2015)

[N3] R. G. Novikov, Phaseless inverse scattering in the one-dimensional case, Eurasian Journal of Mathematical and Computer Applications 3(1), 63-69 (2015)

[N4] R. G. Novikov, Inverse scattering without phase information, Seminaire Laurent Schwartz - EDP et applications (2014-2015), Exp. No16, 13p.

[N5] R. G. Novikov, Multipoint formulas for phase recovering from phaseless scattering data, Journal of Geometric Analysis (to appear), https://hal.archivesouvertes.fr/hal-02119523

[P] V. Palamodov, A fast method of reconstruction for X-ray phase contrast imaging with arbitrary Fresnel number, arXiv:1803.08938v1 (2018)

[S] N. J. Sekeljic , Asymptotic Expansion of Bessel Functions; Applications to Esectromagnetics, Dynamics at the Horsetooth, Vol. 2A, Focussed Issue: Asymptotics and Perturbations, 2010.

[R] V. G. Romanov, Inverse problems without phase information that use wave interference, Sib. Math. J. 59(3), 494-504 (2018) 